\title{
Effect of Dispersing Copper Oxide Nanoparticles to RT-35 filled a Circular Thermal Energy Storage System
}

\author{
Soroush Ebadi*, Mohammadhossein Hajiyan, Syeda Tasnim \\ School of Engineering, University of Guelph, \\ Guelph, Canada \\ *sebadi@uoguelph.ca
}

\author{
Amir Abbas Aliabadi, Shohel Mahmud ${ }^{\psi}$ \\ School of Engineering, University of Guelph, \\ Guelph, Canada \\ *smahmud@uoguelph.ca
}

\begin{abstract}
Phase Change Materials (PCMs) have been widely used in Latent Heat Thermal Energy Storage Systems (LHTES) to store and release thermal energy. However, PCMs suffer from their low thermal conductivities, which leads to longer melting (charging) or solidification (discharging) time. Dispersing highly conductive nanoparticles into PCM is one of the methods to improve the thermal conductivity of the PCM. In this paper, we have experimentally and numerically investigated the effect of dispersing Copper Oxide nanoparticles into RT-35 filled a vertical cylindrical Thermal Energy Storage (TES) system. The TES system is consisted of two vertical cylindrical enclosures, which are attached to a thick acrylic plate. One of the enclosures is filled with pure PCM and the other one is filled with nano-PCM. The setup is insulated from the bottom and isothermally heated from its sides by using a constant temperature bath. To study more about the thermal and velocity fields, melting fraction and stored energy, a numerical simulation is conducted by using COMSOL Multiphysics software. It is concluded that although adding nanoparticles to PCM improves the melting rate, it is lowering the amount of energy stored inside the TES system.
\end{abstract}

\section{Keywords: Thermal energy storage (TES); Latent heat; Phase change material (PCM); nano- PCM}

\section{INTRODUCTION}

The increase in the amount of greenhouse gasses especially Carbon dioxide caused by burning fossil fuels has caused a serious environmental concern known as global warming. On the other hand, the increase in the worldwide population and industrial units have forced energy suppliers to search for alternate energy sources (i.e. renewable energy) and or use of energy efficiently. Thermal Energy Storage (TES) systems have developed with the aim of storing thermal energy effectively and release that energy in the demand time. TES systems are divided into three main groups including: (i) Sensible Heat TES system, (ii) Latent Heat TES system and, (iii) Thermo-Chemical TES system. However, among these three groups, LHTES system is more attractive. LHTES systems use PCMs to store or release thermal energy which offers two main advantages: (i) higher capacity of storing energy (i.e. 5-14 times higher than sensible heat TES) and (ii) smoother temperature fluctuations during the operation [1,2]. A wide variety of PCMs are available with different melting temperatures (Tm) which have been used for both cooling and heating purposes. Paraffin waxes, hydrated salts and organic compounds (e.g. Coconut oil) are the most available types of PCMs. Although PCMs seem to be a proper material for store/release of thermal energy, they suffer from a low thermal conductivity. This disadvantage leads to longer melting/solidification time, which affect the performance of the LHTES system [3]. Several methods have been proposed to enhance the thermal conductivity of PCMs such as (i) dispersing highly conductive nanoparticles into the PCM, which is the main purpose of this study, (ii) inserting metal fins, and (iii) the use of conductive porous medium [4]. Different weight fractions/volume fractions of nanoparticles can be dispersed in to the PCM to increasing its thermal conductivity. However, in practical, sedimentation is an issue caused by using high weight/volume fractions of nanoparticles $[5,6]$. Different configuration of PCM container such as rectangular, cylindrical (both horizontal/vertical), and spherical can be used. Cylindrical can be installed both vertically and horizontally according to a specific application/ occupied space [7].

In this paper, the main aim of study is to investigate the melting process of a nano-PCM filled in a vertical cylindrical enclosure. By surveying the literature, it can be found that a considerable number of researchers have studied the use of vertical cylindrical enclosures filled with nano-PCM. Wu et al. [8] performed a numerical study on the melting process of Paraffin enhanced with Copper nanoparticles filled in a cylindrical enclosure which was heated from the bottom. It was found that the melting rate was $13.1 \%$ improved by using $1 \mathrm{wt}$. 
$\%$ of nanoparticles. Sciacovelli et al. [9] numerically studied the melting of paraffinic PCM with copper nanoparticles. In their investigation, the nano-PCM was isothermally heated by placing an inner tube at the center of cylindrical enclosure. It was reported that by using $4 \%$ volume fraction of nanoparticles, the melting time was decreased by $15 \%$. Das et al. [10] numerically studied the melting process of n-eicosane enhanced with carbon allotropes. The enclosure was isothermally heated placing an inner pipe at the center. It was concluded that by using $1 \%$ volume fraction of single-walled carbon nanotubes, the melting time melting time was decreased by $15 \%$. The melting time was decreased up to $25 \%$ with the use of $1 \%$ volume fraction of graphene. An experimental study on melting process of 1-dodeconal enhanced with carbon nanotubes was performed by Zeng et al. [11]. In their experiment, the cylindrical enclosure was insulated from the surroundings and heated from the bottom. It is reported that by dispersing the carbon nanotubes, melting rate was degraded due to the increase of viscosity and decrease in the natural convection. Fan et al. [12] experimentally investigated the effect of adding Graphene nanoplatelets in to tetradecanol on the melting rate. The cylindrical enclosure was isothermally heated from the bottom. It was reported that by using $3 \mathrm{wt} . \%$ of nanoparticles at the temperature of $55^{\circ} \mathrm{C}$, a $8 \%$ decrease in the melting time is observed.

According to the literature, the melting process of a nanoPCM inside a vertical cylindrical enclosure, insulated from the bottom, and isothermally heated from the sides has not been extensively investigated. In this work, an experimental and numerical investigation are performed to study the effect of adding Copper oxide nanoparticles into RT-35 with the melting temperature $\left(\mathrm{T}_{\mathrm{m}}\right)$ in a range of $34{ }^{\circ} \mathrm{C}-36{ }^{\circ} \mathrm{C}$. To do so, an experimental setup is built to visualize and compare the melting process of pure PCM and nano-PCM. Moreover, a COMSOL simulation is developed to compare the energy stored in PCM and nano-PCM.

\section{EXPERIMENTAL INVESTIGATION}

\section{A. Experimental setup}

The primary objective of the experimental work is to visualize and compare the melting process of PCM and nanoPCM. The experimental setup is consisted of two transparent acrylic pipe with the inner diameter of $4.4 \mathrm{~cm}$, a transparent water bath equipped with temperature regulator, a circulation pump, and a digital camera to capture the melting pattern periodically. Two cylindrical acrylic pipes are vertically attached to a thick plastic plate (thickness $=3.5 \mathrm{~cm}$ ) with the center-to-center distance of $13 \mathrm{~cm}$. The cylindrical pipes are thermally insulated from the bottom and to avoid any pressure at the top of PCM/nano-PCM, it is kept open from the top. A schematic of the experimental setup is shown in Fig.1.

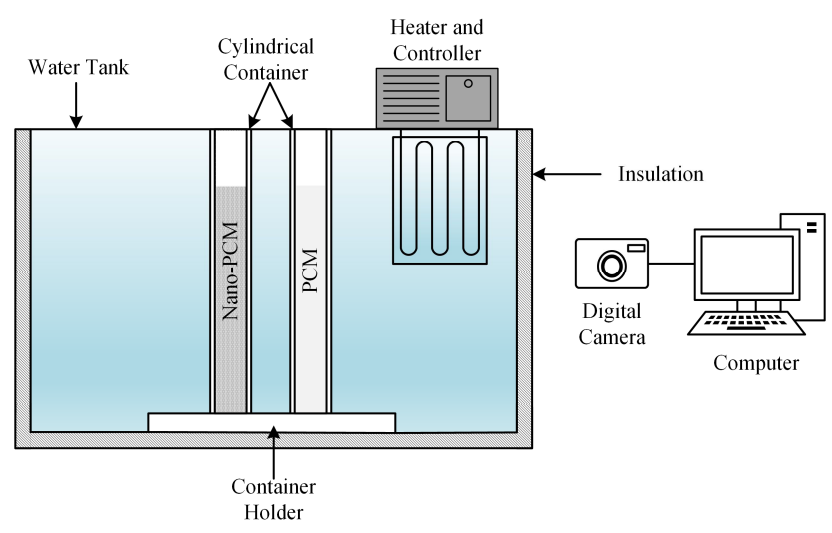

Fig.1. A schematic of the experimental setup

\section{B. Nano-PCM preparation}

One of the main challenges associated with the use of nano-PCM is the sedimentation of nanoparticles at the bottom of enclosure after several cycles of operation. Therefore, preparation of the nano-PCM is an important step for the experimental work. At the first step, the required amount of RT-35 and Copper oxide nanoparticles to have a 0.2 wt. $\%$ nano-PCM was weighed by a precise balance. It should be mentioned that dispersing Copper oxide nanoparticles into RT-35 results to a dark black fluid. Thus, to have a clear melting visualization, low amount of nanoparticles is used. Then, the mixture was heated up to $60{ }^{\circ} \mathrm{C}$ and stirred by using a magnetic stirrer for 24 hours. To ensure the homogeneity of mixture and release the trapped air inside the nano-PCM, an ultrasound sonicator was used for two hours. At the next step, the nano-PCM was kept in a bath with the temperature of $45^{\circ} \mathrm{C}$ for three hours (more than the time required for melting experiment) to see the amount of sedimentation. After ensuring the homogeneity of mixture, the nano-PCM was solidified gradually at the room temperature (i.e., $\mathrm{T}_{0=}$ $23{ }^{\circ} \mathrm{C}$ ). Solidification at the temperatures close to the $\mathrm{T}_{\mathrm{m}}$ requires a longer time, however, it provides the advantages of having less trapped air inside the nanoPCM and avoiding the sub-cooling effect. Table.1 shows the thermophysical properties of RT-35 and Copper Oxide nanoparticles.

Table 1. Properties of RT-35 and $\mathrm{CuO}$ nanoparticles

\begin{tabular}{|c|c|c|}
\hline Property & RT-35 [13] & Copper oxide[14] \\
\hline$\beta(1 / \mathrm{K})$ & $6 \times 10^{-4}$ & $85 \times 10^{-7}$ \\
\hline$h_{s f}(\mathrm{~kJ} / \mathrm{kg})$ & 170 & - \\
\hline$k(\mathrm{~W} / \mathrm{m} \mathrm{K})$ & 0.2 & 18 \\
\hline$\mu(\mathrm{Pa} . \mathrm{s})$ & 0.023 & - \\
\hline$c_{p}(\mathrm{~kJ} / \mathrm{kg} \mathrm{K})$ & 2 & 0.54 \\
\hline$\rho\left(\mathrm{kg} / \mathrm{m}^{3}\right)$ & 815 & 6500 \\
\hline
\end{tabular}




\section{Experimental procedure}

Several steps were taken to start the experiment. First, a predetermined amount of molten Pure PCM and nano-PCM was poured in cylindrical pipes at the same height $(\mathrm{H}=8.3$ $\mathrm{cm})$ representing Rayleigh number of $10^{7}$. Then, the $\mathrm{PCM} /$ nano-PCM were gradually solidified by keeping the setup at the room temperature. Next, the temperature of the constant bath was set to $45^{\circ} \mathrm{C}$. To ensure the accuracy of the thermal regulator, a T-type OMEGA thermocouple was placed inside the bath and monitored the temperature. The Cylindrical TES system enclosures were suddenly placed inside the bath to initiate the melting process. A digital camera was used to record the melting pattern periodically (i.e. every five minutes) during melting process.

\section{NUMERICAL INVESTIGATION}

\section{A. Numerical model}

To study the effect of dispersing nanoparticles to pure PCM on the thermal fields, melting fraction and energy stored, a numerical model with the same geometry as the experimental setup was developed by using COMSOL Multiphysics. The model is insulated from the bottom, isothermally heated from its sides, and filled with PCM/nanoPCM at the height of $\mathrm{H}$. The liquid phase of PCM/nano-PCM is assumed as a Newtonian incompressible fluid. A 2-D view of the numerical model is shown below.

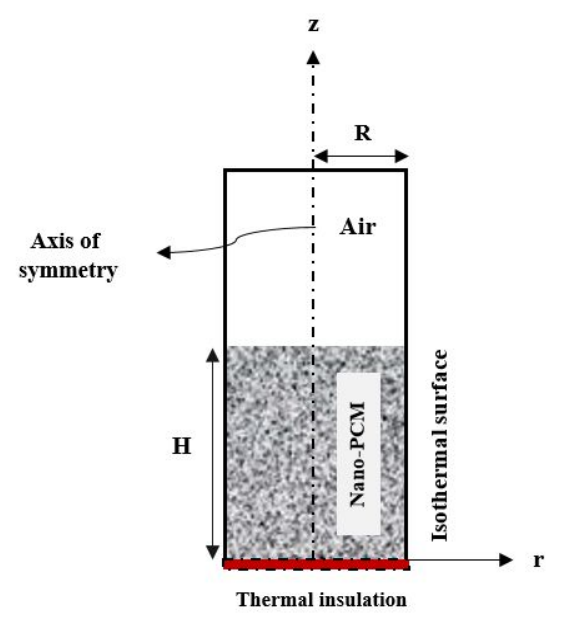

Fig. 2. A 2-D view of the numerical model

The governing equations representing the conservation of mass, momentum and transferred energy are presented below [15]:

$$
\text { Continuity equation : } \frac{\partial u}{\partial r}+\frac{u}{r}+\frac{\partial w}{\partial z}=0
$$

where $u$ and $w$ are the components of velocity in r-direction and $\mathrm{z}$ - direction, respectively.

Energy equation:

$$
\frac{\partial T}{\partial t}+u \frac{\partial T}{\partial r}+w \frac{\partial T}{\partial z}=\frac{k}{\rho C_{p}}\left[\frac{1}{r} \frac{\partial}{\partial r}\left(r \frac{\partial T}{\partial r}\right)+\frac{\partial^{2} T}{\partial z^{2}}\right]
$$

where $t, K, \rho$ and $C_{p}$ represent time, thermal conductivity, density and specific heat at constant pressure, respectively.

Momentum (z-direction):

$$
\frac{\partial w}{\partial t}+u \frac{\partial w}{\partial r}+w \frac{\partial w}{\partial z}=\frac{1}{\rho} \frac{\partial p}{\partial z}+\frac{\mu}{\rho}\left(\frac{\partial^{2} w}{\partial r^{2}}+\frac{1}{r} \frac{\partial w}{\partial r}+\frac{\partial^{2} w}{\partial z^{2}}\right)+g \beta\left(T-T_{m}\right)
$$

Momentum(r-direction):

$$
\frac{\partial u}{\partial t}+u \frac{\partial u}{\partial r}+w \frac{\partial u}{\partial z}=-\frac{1}{\rho} \frac{\partial p}{\partial r}+\frac{\mu}{\rho}\left(\frac{\partial^{2} u}{\partial r^{2}}+\frac{1}{r} \frac{\partial u}{\partial r}-\frac{u}{r^{2}}+\frac{\partial^{2} u}{\partial z^{2}}\right)
$$

where $\mu, g, \beta$ and $T$ represent viscosity, gravitational acceleration and coefficient of thermal expansion and temperature, respectively. The amount of stored energy is one of the main parameters for designing a LTES system which can be determined by Eq. 5 [16].

$$
E=\int_{0}^{t} \dot{Q}(t) d t
$$

In this equation, $\hat{Q}(t)$ is the transferred heat during the melting process which can be determined by Eq. 6 .

$$
\dot{Q}(t)=(\pi d) \int_{0}^{H}\left(-k \frac{\partial T}{\partial r}\right) d z+(2 \pi) \int_{0}^{R}\left(-k \frac{\partial T}{\partial z}\right) r d r
$$

Where $d$ and $k$ are the inner diameter of the circular pipe and thermal conductivity of the nano-PCM respectively.

Dispersion of nanoparticles to PCM results to new properties such as density, viscosity, thermal conductivity, and heat capacity. The thermophysical property relationships for the nano-PCM can be found in [17]. Moreover, as the change in the phase of the nano-PCM occurs within a transition stage, the properties of the nano-PCM are affected by a transition temperature range (i.e. $\mathrm{T}=\mathrm{T}_{\mathrm{m}}-\Delta \mathrm{T}$, and $\mathrm{T}=\mathrm{T}_{\mathrm{m}}+\Delta \mathrm{T}$ ). The thermophysical properties of the nano-PCM at the transition 
stage can be found in [18]. In addition, a Gaussian function is used to approximate the heat capacity during the phase change process [19].

\section{B. Grid size and time step independency test}

To test the mesh size and time intervals independency, simulations were carried out in different steps. First, the melting fraction was calculated by using three different mesh sizes including (i) 6965 elements, (ii) 15062 elements and (iii) 33905 elements. The result of this test is shown in Fig.3.

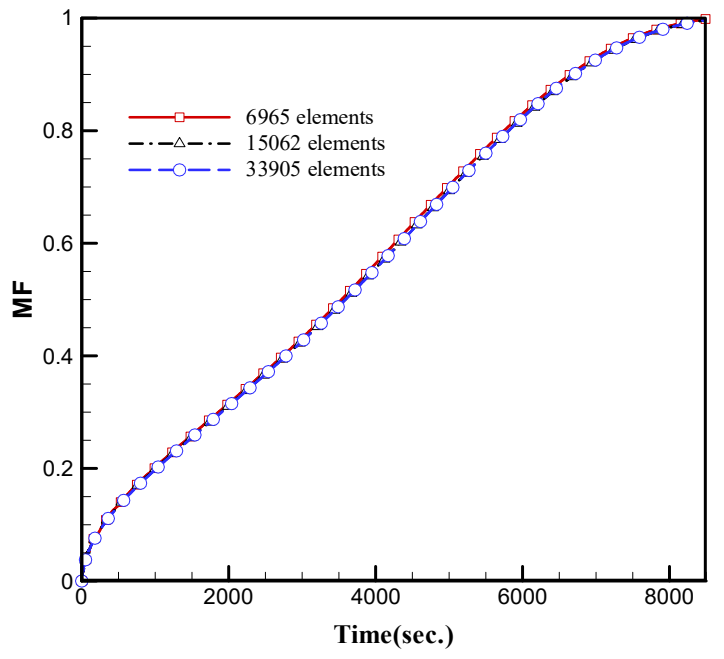

Fig. 3. Grid size independency test

As it can be seen from Fig. 3, the values of melting fraction for different meshes are almost identical. Hence, to obtain the proper accuracy and less simulation time, the mesh quality with 15062 elements was selected. At the next step, the time step independency is examined. In this test, melting fraction was calculated by using three different simulations with different time steps. Table 2 shows the different time steps used in these series of tests.

Table 2. Time intervals used to examine the time step independency

\begin{tabular}{|c|c|c|c|}
\hline \multirow{2}{*}{$\begin{array}{c}\text { Time zone } \\
(\mathrm{s})\end{array}$} & \multicolumn{3}{|c|}{$\begin{array}{c}\text { Time step } \\
\text { (s) }\end{array}$} \\
\cline { 2 - 4 } 0 to 10 & 0.01 & 0.1 & 0.005 \\
\hline 10 to 100 & 0.1 & 1 & 0.05 \\
\hline 100 to 10000 & 10 & 100 & 5 \\
\hline
\end{tabular}

Fig.4 presents the result of these tests. As it can been seen, there is a slight difference in the values of melting fraction resulted by these three time steps. Therefore, to have both accuracy and less simulation time, the simulations were carried out by using the time interval in Test 2.

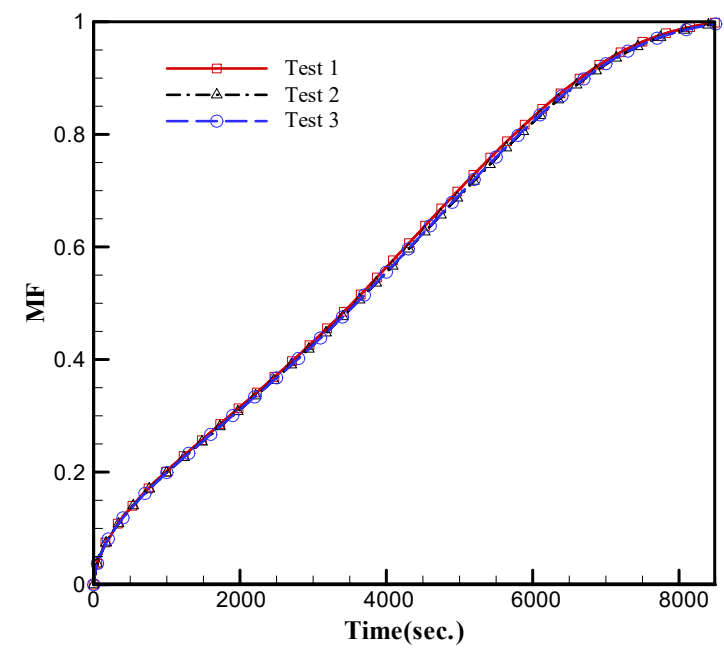

Fig. 4. Time step independency test

\section{Validation with experimental work}

Figure 5 presents a melting visualization comparison between numerical results and experimental work for 0.2 wt.\% of nano-PCM. To do so, some selected results obtained from numerical and experimental are compared. In general, a good agreement is achieved. However, there are some discrepancies in melting pattern, which may be due to the two main factors. First, the air trapped inside the nano-PCM in the experimental work was seen to cause a non-uniform melting at the times that trapped air was released. The other major factor is neglecting of the thermal resistance of the acrylic pipe in the numerical model. As a result, more heat could be transferred between hot wall and the nano-PCM during numerical simulation. 


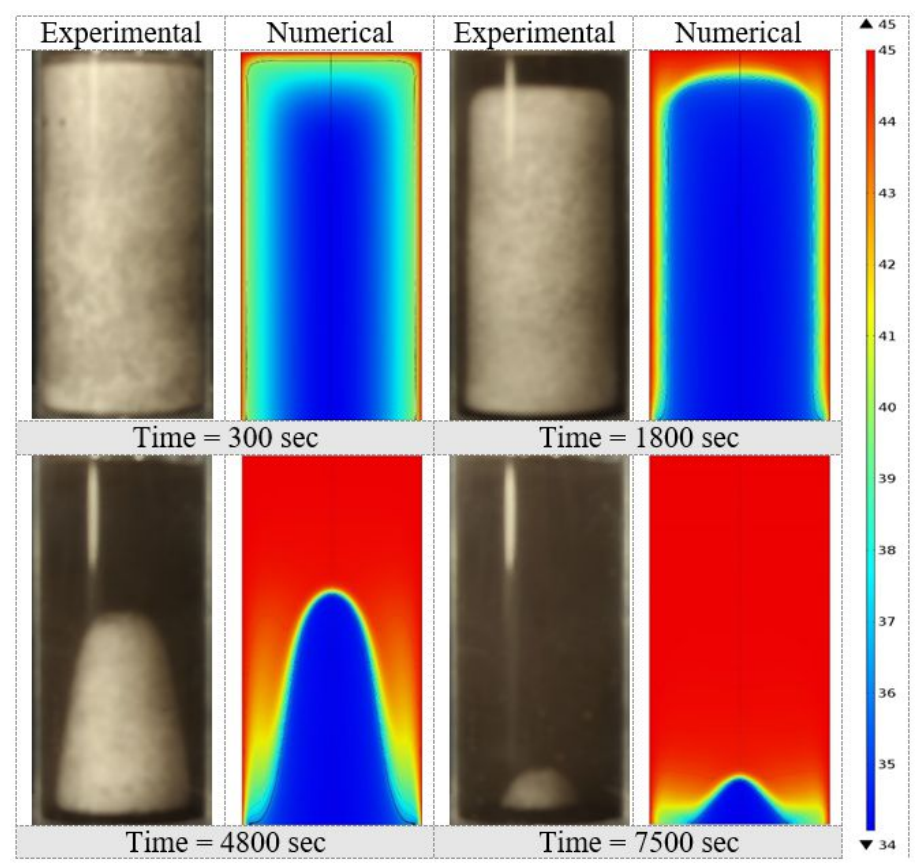

Fig. 5. Validation of numerical model with experimental work

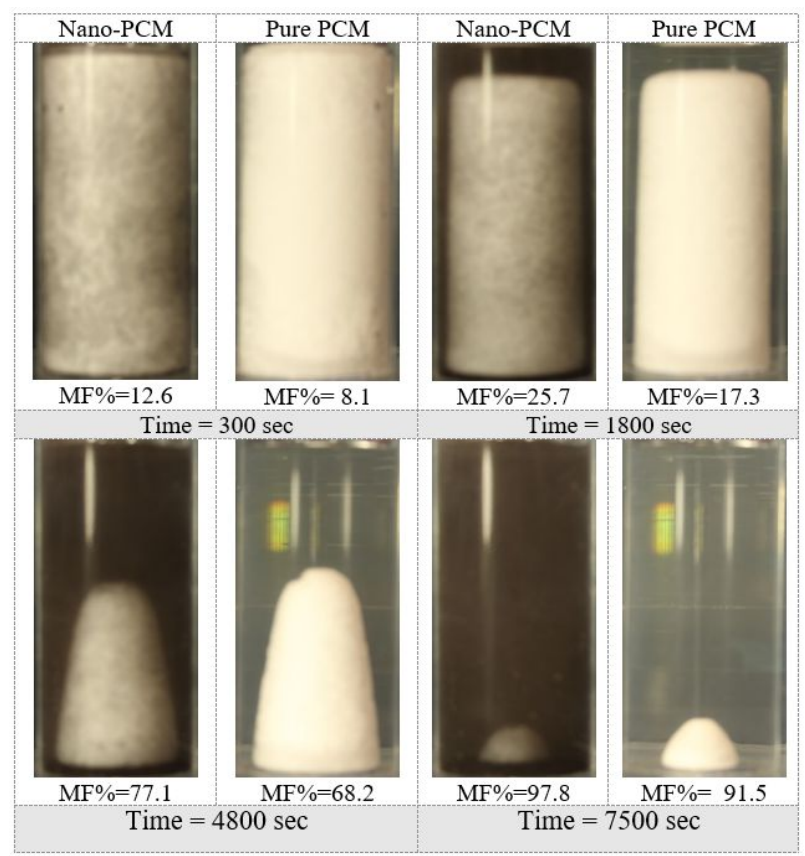

Fig. 6. Visualization of melting pattern for nano-PCM and pure PCM

\section{B. Energy Stored}

\section{A. Melting pattern visualization}

In this section a comparison of melting pattern between pure PCM and Nano-PCM is presented. Some selected results are presented in figure 6 . After a few minutes of initiating the experiment, a thin layer of molten PCM/nano-PCM appeared which lead to a solid-liquid interface. At the early stages of melting process (up to $300 \mathrm{~s}$ ), conduction was the dominant mode of heat transfer since the solid-liquid interface is parallel to the lateral walls. As time furthered, the buoyancy force started to dominate the viscous force and natural convection started to establish. This phenomenon can be clearly observed from the remaining shape of the solid PCM/nanoPCM. At the later stages of melting process (i.e. after 3600s), convection became the dominant mode of heat transfer which resulted to a dome shape solid $\mathrm{PCM} /$ nano-PCM. As it can be seen from the figure 6, an improvement in the melting rate is observed by using 0.2 wt. \% nano-PCM. To assist the discussion, the melting fractions for both PCM and nano-PCM at different times was calculated from the digital photos by using a software called Grafula. Calculation of melting fraction from digital image was also used in other published works in the literature such as [20]. 


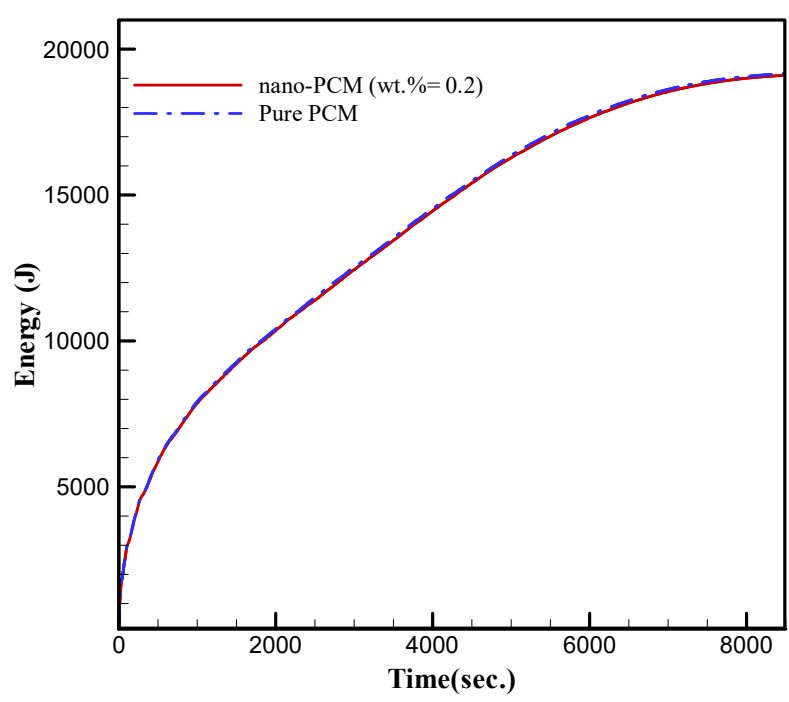

Fig.7. Stored energy

V. CONCLUSION

A numerical and experimental study were conducted to investigate the effect of dispersing copper oxide nanoparticles into the RT-35 as the base PCM on the melting rate and energy stored. The followings can be concluded from the results obtained from this work:

a) Dispersion of the Copper oxide nanoparticles in to the RT-35 increased the melting rate due to enhancing the thermal conductivity of base PCM. From the numerical simulations, it can be found that this improvement increases by using higher weight/volume fractions of the nanoparticles. However, it is worth mentioning that in practical, dispersion of high weight/volume fractions of nanoparticles results to the sedimentation issue.

b) The rate of energy stored is seen to be high at the beginning of melting process due to the high temperature gradient. This stored energy rate degraded to zero at the end of melting process due to the thermal equilibrium. In addition, nano-PCM showed a lower amount of energy stored.

c) Finally, as both melting time (charging) and the total energy stored are important for an optimal design of a LHTES system, it is recommended to maintain a balance between improvement in the thermal conductivity and decrease in the energy stored if use of nano-PCM is required.

\section{REFERENCES}

[1] A. Kumar, S.K. Shukla, A Review on thermal energy storage unit for solar thermal power plant application, Journal of Energy Procedia, 74 (2015) 462 469.

[2] Zalba, J. Marın, L.F. Cabeza, H. Mehling, A review on thermal energy storage with phase change materials: heat transfer analysis and applications, Applied Thermal Engineering, 23(2003) 251-283.

[3] G. Fang, H. Li, F. Yang, X. Liu, S. Wu, Preparation and characterization of nano-encapsulated n-tetradecane as phase change material for thermal energy storage, Journal of Chemical Engineering, 153 (2009) 217-221.

[4] N. Dhaidan, Nanostructures assisted melting of phase change materials in various cavities, Applied Thermal Engineering, 111 (2017) 193-212.

[5] X. Zhang, X. Chen, Z. Han, W. Xu, Study on phase change interface for erythritol with nano-copper in spherical container during heat transport, International Journal of Heat and Mass Transfer, 92 (2016) 490-496.

[6] R.K. Sharma, P. Ganesan, V.V. Tyagi, H.S.C. Metselaar, S.C. Sandaran, Thermal properties and heat storage analysis of palmitic acid-TiO2 composite as nano-enhanced organic phase change material (NEOPCM), Applied Thermal Engineering, 99 (2016) 1254-1262.

[7] N. Dhaidan, J. Khodadadi, Melting and convection of phase change materials in different shape containers: A review, Journal of Renewable and Sustainable Energy Reviews, 43 (2015) 449-477.

[8] S. Wu, H. Wang, S. Xia, D. Zhu, Numerical simulation on thermal energy storage behavior of cu/paraffin nanofluids PCMs, Procedia Engineering, 31 (2012) 240-244.

[9] A. Sciacovelli, F. Colella, V. Verda, Melting of PCM in a thermal energy storage unit: Numerical investigation and effect of nanoparticle enhancement, International Journal of Energy Research, 37 (2013) 1610-1623.

[10] N. Das, Y. Takata, M. Kohno, S. Harish, Effect of carbon nano inclusion dimensionality on the melting of phase change nanocomposites in vertical shell-tube thermal energy storage unit, International Journal of Heat and Mass Transfer, 113 (2017) 423-432.

[11] Y. Zeng, L. Fan, Y. Xiao, Z. Yu, K. Cen, An experimental investigation of melting of nanoparticle-enhanced phase change materials (NePCMs) in a bottom heated vertical cylindrical cavity, International Journal of Heat and Mass Transfer, 66 (2013) 111-117.

[12] L. Fan, Z. Zhu, Y. Zeng, Q. Lu, Z. Yu, Heat transfer during melting of graphene based composite phase change materials heated from below, International Journal of Heat and Mass Transfer, 79 (2014) 94-104.

[13] M. Esapour, M.J. Hosseini, A.A. Ranjbar, R. Bahrampoury, Numerical study on geometrical specifications and operational parameters of multi-tube heat storage systems. Applied Thermal Engineering, 109, 351-363.

[14] Choi, S. Kim, T. Lee, D. Hahn, Computation of the natural convection of nanofluid in a squared cavity with homogeneous and nonhomogeneous models, Numerical heat transfer, 65 (2014) 287-301.

[15] A. Bejan, Convection Heat Transfer, Wiley, New York, 2013.

[16] C.J. Ho, J.Y. Gao, An experimental study on melting heat transfer of paraffin dispersed with $\mathrm{Al}_{2} \mathrm{O}_{3}$ nanoparticles in a vertical enclosure, International Journal of Heat and Mass Transfer, 62 (2013) 2-8.

[17] J.M. Khodadadi, S.F. Hosseinzadeh, Nanoparticle-enhanced phase change materials (NEPCM) with great potential for improved thermal energy storage, International Communications in Heat and Mass Transfer 34 (2007) 534-543.

[18] N.S. Dhaidan, J.M. Khodadadi, T.A. Al-Hattab and S.M. Al-Mashat, Experimental and numerical investigation of melting of phase change material/nanoparticle suspensions in a square container subjected to a constant heat flux, International Journal of Heat and Mass Transfer, 66 (2013) 672-683. [19] D. Groulx, "Numerical study of nano-enhanced PCMs: Are they worth it?" Proceedings of the 1st Thermal and Fluid Engineering Summer Conference, TFESC August 9-12, 2015, New York City, USA

[20] F.L. Tan, Constrained and unconstrained melting inside a sphere, International Communications in Heat and Mass Transfer, 35 (2008) 466-475. 